International Journal of Pure and Applied Mathematics

Volume 85 No. 1 2013, 179-191

ISSN: 1311-8080 (printed version); ISSN: 1314-3395 (on-line version)

url: http://www.ijpam.eu

doi: http://dx.doi.org/10.12732/ijpam.v85i1.15

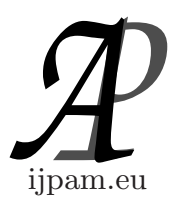

\title{
SUMS OF THE RATIONAL POWERS OF ROOTS OF THE POLYNOMIALS
}

\author{
Roman Wituła ${ }^{1 \S}$, Edyta Hetmaniok ${ }^{2}$, \\ Damian Słota $^{3}$, Natalia Gawrońska ${ }^{4}$ \\ 1,2,3,4 Institute of Mathematics \\ Silesian University of Technology \\ Kaszubska 23, 44-100 Gliwice, POLAND
}

\begin{abstract}
In this paper the completely elementary methods of generating the trigonometric identities are presented. These methods are based on the discussion of sums of the rational powers of roots of the polynomials. Among investigated identities there are proven the identities connected with roots of Kepler's polynomial and generalizations of the known Ramanujan's equalities.
\end{abstract}

AMS Subject Classification: 11B83, 11Y55

Key Words: sums of the roots, Ramanujan's formulae

\section{Introduction}

The current paper is a supplement of the authors' previous work [13].

Similarly as in paper [13] the direct reason of preparing the present work was a trial of the pure algebraic look on the following excellent Ramanujan formulae

$$
\left(\frac{1}{9}\right)^{1 / 3}-\left(\frac{2}{9}\right)^{1 / 3}+\left(\frac{4}{9}\right)^{1 / 3}=(\sqrt[3]{2}-1)^{1 / 3}
$$

Received: $\quad$ March 13, 3013

(c) 2013 Academic Publications, Ltd.

$\S_{\text {Correspondence author }}$ 


$$
\begin{aligned}
& \left(\cos \frac{2 \pi}{7}\right)^{1 / 3}+\left(\cos \frac{4 \pi}{7}\right)^{1 / 3}+\left(\cos \frac{8 \pi}{7}\right)^{1 / 3}=\left(\frac{5-3 \sqrt[3]{7}}{2}\right)^{1 / 3} \\
& \left(\cos \frac{2 \pi}{9}\right)^{1 / 3}+\left(\cos \frac{4 \pi}{9}\right)^{1 / 3}+\left(\cos \frac{8 \pi}{9}\right)^{1 / 3}=\left(\frac{3 \sqrt[3]{9}-6}{2}\right)^{1 / 3}
\end{aligned}
$$

together with the identities inspired by formulae (1)-(2) and derived by the authors in recent papers $[9,10,11]$, like for example:

$$
\begin{gathered}
\sqrt[3]{\frac{\cos (\boldsymbol{\beta})}{\cos (4 \beta)}}(2 \cos (\boldsymbol{\beta}))^{n}+\sqrt[3]{\frac{\cos (2 \boldsymbol{\beta})}{\cos (\boldsymbol{\beta})}}(2 \cos (2 \boldsymbol{\beta}))^{n}+ \\
+\sqrt[3]{\frac{\cos (4 \boldsymbol{\beta})}{\cos (2 \boldsymbol{\beta})}}(2 \cos (4 \boldsymbol{\beta}))^{n}=-\left(\sqrt[3]{\frac{\cos (2 \boldsymbol{\beta})}{\cos (\boldsymbol{\beta})}}(2 \cos (\boldsymbol{\beta}))^{n+1}+\right. \\
\left.+\sqrt[3]{\frac{\cos (4 \boldsymbol{\beta})}{\cos (2 \boldsymbol{\beta})}}(2 \cos (2 \boldsymbol{\beta}))^{n+1}+\sqrt[3]{\frac{\cos (\boldsymbol{\beta})}{\cos (4 \boldsymbol{\beta})}}(2 \cos (4 \boldsymbol{\beta}))^{n+1}\right)= \\
=-\left(\sqrt[3]{2 \cos (2 \boldsymbol{\beta})(2 \cos (\boldsymbol{\beta}))^{3 n+2}}+\sqrt[3]{2 \cos (4 \boldsymbol{\beta})(2 \cos (2 \boldsymbol{\beta}))^{3 n+2}}+\right. \\
\left.+\sqrt[3]{2 \cos (\boldsymbol{\beta})(2 \cos (4 \boldsymbol{\beta}))^{3 n+2}}\right)=\sqrt[3]{9} \Psi_{n},
\end{gathered}
$$

where $\beta=\frac{2 \pi}{9}, \Psi_{0}=-1, \Psi_{1}=1, \Psi_{2}=-4$ and

$$
\Psi_{n+3}-3 \Psi_{n+1}+\Psi_{n}=0, \quad n \in \mathbb{Z} .
$$

It is obvious that each of relations (4) is the Binet formula for the respective recurrence relation (5). Problem of deriving formulae of type (4) reduces in practice to decomposing the respective characteristic polynomials and finding the initial values. Especially the latter is a fundamental problem. In the current paper we present some technical tools enabling to generate the formulae of type (4) in specifically elementary way. Results of using these tools will be presented on the particular examples and seem to be equally attractive.

\section{Basic Theorems}

We present now our crucial technical results. Proofs of all theorems are given in paper [13].

Let $f \in \mathbb{C}[\mathbb{X}]$ be such that

$$
f(\mathbb{X})=\mathbb{X}^{3}+p \mathbb{X}^{2}+q \mathbb{X}+r=\left(\mathbb{X}-\xi_{1}\right)\left(\mathbb{X}-\xi_{2}\right)\left(\mathbb{X}-\xi_{3}\right) .
$$


Theorem 1 (simple version). If $p \in 3 \sqrt[3]{r}$, then the values $\alpha, \beta, \gamma$ of complex roots $\sqrt[3]{\xi_{1}}, \sqrt[3]{\xi_{2}}, \sqrt[3]{\xi_{3}}$, respectively, can be chosen in such a way that

$$
\alpha+\beta+\gamma=0 .
$$

In the sequel, if $\xi_{1}, \xi_{2}, \xi_{3}$ are the real numbers, then we can assume that also $\alpha, \beta, \gamma$ are the real numbers.

Let us present now the following version of Theorem 1 which holds for more general family of cubic polynomials.

Theorem 2 (general version). If $3 q \neq p^{2}$ or if $3 q=p^{2}$ and $27 r=p^{3}$, then there exists $c \in \mathbb{C}$ such that, for the respective $\alpha \in \sqrt[3]{\xi_{1}+c}, \beta \in \sqrt[3]{\xi_{2}+c}$ and $\gamma \in \sqrt[3]{\xi_{3}+c}$, we have

$$
\alpha+\beta+\gamma=0 .
$$

If $\xi_{1}, \xi_{2}, \xi_{3} \in \mathbb{R}$ and $3 q \neq p^{2}$, then the above equality holds for

$$
c=\frac{p^{3}-27 r}{9\left(p^{2}-3 q\right)}
$$

(all roots should be real). Whereas, if $3 q=p^{2}$ and $27 r \neq p^{3}$, then such constant $c \in \mathbb{C}$ does not exist.

Let $d \in \mathbb{R}$. If $\xi_{1}, \xi_{2}, \xi_{3} \in \mathbb{R}$, then the following formula holds true

$$
\begin{aligned}
\sqrt[3]{\xi_{1}+d}+\sqrt[3]{\xi_{2}+d}+ & \sqrt[3]{\xi_{3}+d} \\
& =\sqrt[3]{-P-6 \sqrt[3]{R}-\frac{3}{\sqrt[3]{2}}(\sqrt[3]{\mathcal{S}+\sqrt{\mathcal{T}}}+\sqrt[3]{\mathcal{S}-\sqrt{\mathcal{T}}})}
\end{aligned}
$$

where

$$
\begin{aligned}
\mathcal{S}:= & P Q+6 Q \sqrt[3]{R}+6 P \sqrt[3]{R^{2}}+9 R \\
\mathcal{T}:= & P^{2} Q^{2}-4 Q^{3}-4 P^{3} R+18 P Q R-27 R^{2}, \\
& \prod_{k=1}^{3}\left(\mathbb{X}-\xi_{k}-d\right)=\mathbb{X}^{3}+P \mathbb{X}^{2}+Q \mathbb{X}+R,
\end{aligned}
$$

and

$$
\begin{aligned}
& P=p-3 d \\
& Q=q+3 d^{2}-2 d p \\
& R=r-d^{3}+p d^{2}-q d
\end{aligned}
$$


Additionally, we have

$$
\begin{aligned}
\sqrt[3]{\xi_{1}^{2}}+\sqrt[3]{\xi_{2}^{2}}+\sqrt[3]{\xi_{3}^{2}} \\
\quad=\sqrt[3]{-p_{2}-6 \sqrt[3]{r_{2}}-\frac{3}{\sqrt[3]{2}}\left(\sqrt[3]{\mathcal{S}_{2}+\sqrt{\mathcal{T}_{2}}}+\sqrt[3]{\mathcal{S}_{2}-\sqrt{\mathcal{T}_{2}}}\right)}
\end{aligned}
$$

where

$$
\begin{aligned}
& \mathcal{S}_{2}:=p_{2} q_{2}+6 q_{2} \sqrt[3]{r_{2}}+6 p_{2} \sqrt[3]{r_{2}^{2}}+9 r_{2}, \\
& \mathcal{T}_{2}:=p_{2}^{2} q_{2}^{2}-4 q_{2}^{3}-4 p_{2}^{3} r_{2}+18 p_{2} q_{2} r_{2}-27 r_{2}^{2}, \\
& p_{2}=2 q-p^{2} \\
& q_{2}=q^{2}-2 p r \\
& r_{2}=-r^{2}
\end{aligned}
$$

In the next theorem we present the recurrence sequence which is important for the further discussion.

Theorem 3 (special case of the Newton-Girard formula). If for the certain values $\alpha \in \xi_{1}^{1 / 3}, \beta \in \xi_{2}^{1 / 3}, \gamma \in \xi_{3}^{1 / 3}$ we have

$$
\alpha+\beta+\gamma=0
$$

and we set

$$
S_{n}:=\alpha^{n}+\beta^{n}+\gamma^{n}, \quad n=0,1,2, \ldots,
$$

then from the Newton-Girard formula (see Remark 1 in [10]) we get

$$
S_{n+3}=\alpha \beta \gamma S_{n}+\frac{1}{2} S_{2} S_{n+1}, \quad n=0,1,2, \ldots
$$

Here we have $\alpha \beta \gamma \in-\sqrt[3]{r}$, whereas $S_{2}$ belongs to the right side of (7) for the respective values of all five complex roots appearing in this root of the third order (both $\sqrt{\mathcal{T}}$ are chosen with the same value).

In the sequel, if $\xi_{1}, \xi_{2}, \xi_{3} \in \mathbb{R}$ and $\mathcal{T}_{2} \geq 0$, then we can assume that all the above roots are real.

Remark 4. More information about generalizations of sequence $S_{n}$, its importance and its properties can be found in paper [3]. 


\section{Application of Fundamental Theorems}

Similarly as in paper [13] we want to give many specific examples of applications of the indicated theorems. In other words, we present several examples of polynomials with the known prime factors decompositions to which our fundamental theorems will be applied. It will lead us to generate new original equalities and identities, of trigonometric nature especially. Collection of these examples completes the appropriate collection given in paper [13]. It will be also used in our activity within the framework of OEIS-Wiki. Henceforward we will denote

$$
\boldsymbol{\alpha}=\frac{2 \pi}{7} \quad \text { and } \quad \boldsymbol{\beta}=\frac{2 \pi}{9} .
$$

$1^{0}$ We have the following decomposition

$$
\mathbb{X}^{3}+\mathbb{X}^{2}-2 \mathbb{X}-1=\prod_{k=0}^{2}\left(\mathbb{X}-2 \cos \left(2^{k} \boldsymbol{\alpha}\right)\right) .
$$

Hence we also receive

$$
\mathbb{X}^{3}-3 \mathbb{X}^{2}-46 \mathbb{X}-1=\prod_{k=0}^{2}\left(\mathbb{X}-\frac{\cos \left(2^{k} \boldsymbol{\alpha}\right)}{\cos \left(2^{k+1} \boldsymbol{\alpha}\right)}\left(2 \cos \left(2^{k} \boldsymbol{\alpha}\right)\right)^{3}\right)
$$

which, by Theorem 1, implies

$$
\sum_{k=0}^{2} \sqrt[3]{\frac{\cos \left(2^{k} \boldsymbol{\alpha}\right)}{\cos \left(2^{k+1} \boldsymbol{\alpha}\right)}} \cos \left(2^{k} \boldsymbol{\alpha}\right)=0
$$

Furthermore, from Theorem 3 it can be generated the formula

$$
S_{n+3}=\sqrt[3]{49} S_{n+1}+S_{n},
$$

where for $n=0,1,2, \ldots$ :

$$
\begin{aligned}
S_{n}:=\sum_{k=0}^{2}\left(\sqrt[3]{\frac{\cos \left(2^{k} \boldsymbol{\alpha}\right)}{\cos \left(2^{k+1} \boldsymbol{\alpha}\right)}} 2 \cos \left(2^{k} \boldsymbol{\alpha}\right)\right)^{n}=\left(\sqrt[3]{\frac{\cos \left(\frac{\pi}{7}\right)}{\cos \left(\frac{2 \pi}{7}\right)}} 2 \cos \left(\frac{\pi}{7}\right)\right)^{n} \\
+\left(-\sqrt[3]{\frac{\cos \left(\frac{2 \pi}{7}\right)}{\cos \left(\frac{3 \pi}{7}\right)}} 2 \cos \left(\frac{2 \pi}{7}\right)\right)^{n}+\left(-\sqrt[3]{\frac{\cos \left(\frac{3 \pi}{7}\right)}{\cos \left(\frac{\pi}{7}\right)}} 2 \cos \left(\frac{3 \pi}{7}\right)\right)^{n}
\end{aligned}
$$

One can also obtain the following decomposition (which can be proven by induction):

$$
S_{n}=\widetilde{a}_{n}+\widetilde{b}_{n} \sqrt[3]{7}+\widetilde{c}_{n} \sqrt[3]{49}, \quad n=0,1,2, \ldots
$$


where, by (11), we have

$$
\begin{aligned}
& \widetilde{a}_{n+3}=\widetilde{a}_{n}+7 \widetilde{b}_{n+1}, \quad \widetilde{b}_{n+3}=7 \widetilde{c}_{n+1}+\widetilde{b}_{n}, \quad \widetilde{c}_{n+3}=\widetilde{a}_{n+1}+\widetilde{c}_{n}, \\
& \tilde{a}_{0}=3, \quad \tilde{a}_{1}=0, \quad \tilde{a}_{2}=0, \quad \tilde{a}_{3}=3, \quad \tilde{a}_{4}=0, \quad \tilde{a}_{5}=0, \\
& \tilde{b}_{0}=0, \quad \tilde{b}_{1}=0, \quad \widetilde{b}_{2}=0, \quad \widetilde{b}_{3}=0, \quad \widetilde{b}_{4}=14, \quad \widetilde{b}_{5}=0, \\
& \widetilde{c}_{0}=0, \quad \widetilde{c}_{1}=0, \quad \widetilde{c}_{2}=2, \quad \widetilde{c}_{3}=0, \quad \widetilde{c}_{4}=0, \quad \widetilde{c}_{5}=5,
\end{aligned}
$$

which implies, after simple calculation, the following recurrence relation

$$
A_{n+9}-3 A_{n+6}-46 A_{n+3}-A_{n}=0
$$

for every $A \in\{a, b, c\}$ and $n \in \mathbb{N}_{0}$. Hence and from the respective initial conditions we deduce that

$$
\begin{aligned}
& a_{n} \neq 0 \quad \Longleftrightarrow 3 \mid n, \\
& b_{n} \neq 0 \quad \Longleftrightarrow n \geq 4 \text { and } 3 \mid(n+2), \\
& c_{n} \neq 0 \quad \Longleftrightarrow 3 \mid(n+1),
\end{aligned}
$$

and

$$
S_{3 n}=a_{3 n}, \quad S_{3 n+1}=b_{3 n+1} \sqrt[3]{7}, \quad S_{3 n+2}=c_{3 n+2} \sqrt[3]{49}
$$

Let us notice that relation (12) holds also for $A_{n}:=S_{n}$.

$2^{0}$ We have decomposition of the form (see [7]):

$$
\mathbb{X}^{3}-\sqrt{7} \mathbb{X}^{2}+\sqrt{7}=\prod_{k=0}^{2}\left(\mathbb{X}-2 \sin \left(2^{k} \boldsymbol{\alpha}\right)\right)
$$

Thus, the equality $\mathbb{X}^{3}-\sqrt{7} \mathbb{X}^{2}+\sqrt{7}=0$ implies that

$$
\mathbb{X}^{3}=\sqrt{7}\left(\mathbb{X}^{2}-1\right)
$$

which concludes

$$
\sqrt[3]{7}=\sum_{k=0}^{2} \sqrt[3]{4 \sin ^{2}\left(2^{k} \boldsymbol{\alpha}\right)-1}=\sum_{k=0}^{2} \sqrt[3]{1-2 \cos \left(2^{k} \boldsymbol{\alpha}\right)}
$$

On the other hand, from (14) we obtain

$$
\begin{aligned}
8 \sin ^{3}\left(2^{k} \boldsymbol{\alpha}\right) & =\sqrt{7}\left(3 \sin ^{2}\left(2^{k} \boldsymbol{\alpha}\right)-\cos ^{2}\left(2^{k} \boldsymbol{\alpha}\right)\right), \\
8 \sin \left(2^{k} \boldsymbol{\alpha}\right) & =\sqrt{7}\left(3-\cot ^{2}\left(2^{k} \boldsymbol{\alpha}\right)\right),
\end{aligned}
$$


for every $k=0,1,2$, which implies

$$
4 \sum_{k=0}^{2} 2 \sin \left(2^{k} \boldsymbol{\alpha}\right)=4 \sqrt{7}=\sqrt{7}\left(9-\sum_{k=0}^{2} \cot ^{2}\left(2^{k} \boldsymbol{\alpha}\right)\right),
$$

i.e.

$$
5=\sum_{k=0}^{2} \cot ^{2}\left(2^{k} \boldsymbol{\alpha}\right)
$$

Moreover, from (13) and (6) we get

$$
\sum_{k=0}^{2} \sqrt[3]{9 \sqrt{7} \sin \left(2^{k} \boldsymbol{\alpha}\right)-17}=0 .
$$

$3^{0}$ From (13) we receive

$$
\mathbb{X}^{3}-7 \mathbb{X}-7=\prod_{k=0}^{2}\left(\mathbb{X}+\frac{\sqrt{7}}{2} \csc \left(2^{k} \boldsymbol{\alpha}\right)\right)
$$

which implies by (6):

$$
\sum_{k=0}^{2} \sqrt[3]{1-\frac{\sqrt{7}}{2} \csc \left(2^{k} \boldsymbol{\alpha}\right)}=0
$$

$4^{0}$ Additionally, from (13) we can obtain the Johannes Kepler polynomial of the form

$$
\mathbb{X}^{3}-7 \mathbb{X}^{2}+14 \mathbb{X}-7=\prod_{k=0}^{2}\left(\mathbb{X}-4 \sin ^{2}\left(2^{k} \boldsymbol{\alpha}\right)\right)
$$

(numbers $4 \sin ^{2}\left(2^{k} \boldsymbol{\alpha}\right), k=0,1,2$, are equal to the squares of chords $A_{1} A_{2}, A_{1} A_{3}$ and $A_{1} A_{4}$ of the regular heptagon $A_{1} A_{2} \ldots A_{7}$ inscribed into the unit circle - see for example $[1,2])$.

Next, from the equality $\mathbb{X}^{3}-7 \mathbb{X}^{2}+14 \mathbb{X}-7=0$ we receive

$$
\mathbb{X}^{3}=7(\mathbb{X}-1)^{2}
$$

which leads to (compare with equality (15)):

$$
\sqrt[3]{49}=\sum_{k=0}^{2} \sqrt[3]{\left(4 \sin ^{2}\left(2^{k} \boldsymbol{\alpha}\right)-1\right)^{2}}=\sum_{k=0}^{2} \sqrt[3]{\left(1-2 \cos \left(2^{k} \boldsymbol{\alpha}\right)\right)^{2}}
$$


Remark 5. We note that (compare with (19)):

$$
\mathbb{X}^{3}-7 \mathbb{X}^{2}+14 \mathbb{X}-\mathbf{8}=(\mathbb{X}-1)(\mathbb{X}-2)(\mathbb{X}-4)
$$

which implies by (6):

$$
\sqrt[3]{1-\frac{127}{63}}+\sqrt[3]{2-\frac{127}{63}}+\sqrt[3]{4-\frac{127}{63}}=0
$$

But the terms are indeed equal to $\frac{\sqrt{ }}{3} \frac{4}{63}, \frac{\sqrt{3}}{3} \frac{1}{63}$ and $\frac{\sqrt{5}}{3} \frac{}{63}$, respectively. So, it is not the interesting case.

Remark 6. From (15) and (20) we get

$$
\prod_{k=0}^{2}\left(\mathbb{X}-\sqrt[3]{1-2 \cos \left(2^{k} \boldsymbol{\alpha}\right)}\right)=\mathbb{X}^{3}-\sqrt[3]{7} \mathbb{X}^{2}+1
$$

since $(a+b+c)^{2}=a^{2}+b^{2}+c^{2}+2(a b+a c+b c)$ and

$$
(a+b+c)^{2}=a^{2}+b^{2}+c^{2}, \quad a b c \stackrel{(8)}{=}-1,
$$

for $a=\sqrt[3]{1-2 \cos (\boldsymbol{\alpha})}, b=\sqrt[3]{1-2 \cos (2 \boldsymbol{\alpha})}, c=\sqrt[3]{1-2 \cos (4 \boldsymbol{\alpha})}$. Moreover we have

$$
4=\sum_{k=0}^{2}\left(1-2 \cos \left(2^{k} \boldsymbol{\alpha}\right)\right)
$$

The following formula can be easily derived

$$
\sum_{k=0}^{2}\left(1-2 \cos \left(2^{k} \boldsymbol{\alpha}\right)\right)^{n / 3}=a_{n}+b_{n} \sqrt[3]{7}+c_{n} \sqrt[3]{49}
$$

for $n=1, \ldots, 100$, where $a_{n}, b_{n}, c_{n} \in \mathbb{Z}$ and

$$
\begin{array}{lll}
a_{n} \neq 0 & \text { only for } & 3 \mid n, \\
b_{n} \neq 0 & \text { only when } & 3 \mid(n+2), \\
c_{n} \neq 0 & \text { only when } & 3 \mid(n+1),
\end{array}
$$

(numerically tested).

$5^{0}$ We have decomposition of the form (see [7]):

$$
\mathbb{X}^{3}-3 \mathbb{X}+\sqrt{3}=(\mathbb{X}-2 \sin (\boldsymbol{\beta}))(\mathbb{X}+2 \sin (2 \boldsymbol{\beta}))(\mathbb{X}-2 \sin (4 \boldsymbol{\beta}))
$$


Hence, we obtain by (6):

$$
\sqrt[3]{6 \sin (\beta)-\sqrt{3}}+\sqrt[3]{6 \sin (4 \beta)-\sqrt{3}}=\sqrt[3]{6 \sin (2 \beta)+\sqrt{3}}
$$

Thus, by Theorems 2 and 3, the formula can be genareted

$$
S_{n+3}=\sqrt[3]{3} S_{n+1}-\frac{\sqrt{3}}{3} S_{n}, \quad n=0,1,2, \ldots,
$$

where

$$
\begin{aligned}
S_{n}:=\left(2 \sin (\boldsymbol{\beta})-\frac{\sqrt{3}}{3}\right)^{n / 3}+\left(-2 \sin (2 \boldsymbol{\beta})-\frac{\sqrt{3}}{3}\right)^{n / 3} & \\
& +\left(2 \sin (4 \boldsymbol{\beta})-\frac{\sqrt{3}}{3}\right)^{n / 3} .
\end{aligned}
$$

In the current paper the further discussion of this sequence will be omitted.

Furthermore, from the equality $\mathbb{X}^{3}-3 \mathbb{X}+\sqrt{3}=0$ we get

$$
\left(\mathbb{X}^{2}-\frac{3}{2}\right)^{2}=\frac{9}{4}-\sqrt{3} \mathbb{X}
$$

which implies

$$
\sqrt{1-\frac{8}{9} \sqrt{3} \sin (\boldsymbol{\beta})}+\sqrt{1+\frac{8}{9} \sqrt{3} \sin (2 \boldsymbol{\beta})}=1+\sqrt{1-\frac{8}{9} \sqrt{3} \sin (4 \boldsymbol{\beta})}
$$

(compare with the equality

$$
\sqrt{1-\frac{8}{9} \cos (\beta)}+\sqrt{1-\frac{8}{9} \cos (4 \beta)}=1+\sqrt{1-\frac{8}{9} \cos (2 \beta)},
$$

given in [13]).

\section{Generalizations}

All the previously discussed problems can be easily generalized to the polynomials of degree higher than 3. For example, the following result holds true. 
Theorem 7. Let $p, q, r \in \mathbb{C}$ and suppose that $\left(\frac{p}{4}\right)^{4}=r$.

If we set

$$
f(\mathbb{X})=\mathbb{X}^{4}+p \mathbb{X}^{3}+\frac{3}{8} p^{2} \mathbb{X}^{2}+q \mathbb{X}+r=\prod_{k=1}^{4}\left(\mathbb{X}-\xi_{k}\right)
$$

then there exist $\alpha_{k} \in \sqrt[4]{\xi_{k}}, k=1, \ldots, 4$, such that

$$
\sum_{k=1}^{4} \alpha_{k}=0
$$

Proof. We propose the following sketch of proof. We note that

$$
f(\mathbb{X})=0 \quad \Longleftrightarrow \quad\left(\mathbb{X}+\frac{p}{4}\right)^{4}+\left(q-\frac{p^{3}}{16}\right) \mathbb{X}=0
$$

which implies

$$
\xi_{k}+\frac{p}{4} \in \sqrt[4]{\left(\frac{p^{3}}{16}-q\right) \xi_{k}}, \quad k=1, \ldots, 4
$$

i.e.

$$
0=p-p=p+\sum_{k=1}^{4} \xi_{k} \in \sum_{k=1}^{4} \sqrt[4]{\left(\frac{p^{3}}{16}-q\right) \xi_{k}}
$$

and

$$
0 \in \sum_{k=1}^{4} \sqrt[4]{\xi_{k}}
$$

Example 8. Let us set

$$
\begin{aligned}
f_{3}(\mathbb{X})=\mathbb{X}^{4}+ & \left(\frac{5}{3} \mathbb{X}-\frac{8}{3}\right)^{3} \\
& =(\mathbb{X}-1)(\mathbb{X}+8)\left(\mathbb{X}-\frac{8}{27}(4-i \sqrt{11})\right)\left(\mathbb{X}-\frac{8}{27}(4+i \sqrt{11})\right)
\end{aligned}
$$

Hence we receive

$$
f_{3}(\mathbb{X})=0 \quad \Longleftrightarrow \quad \mathbb{X}^{4}=\left(\frac{8}{3}-\frac{5}{3} \mathbb{X}\right)^{3}
$$


which implies the relations

$$
\left(\frac{1}{2}(7+i 5 \sqrt{11})\right)^{3}+\left(\frac{1}{2}(7-i 5 \sqrt{11})\right)^{3}=(4-i \sqrt{11})^{4}+(4+i \sqrt{11})^{4}
$$

and

$$
-\left(\frac{5}{3}\right)^{3}=\alpha+\beta+\gamma+\delta
$$

for certain complex values $\alpha \in \sqrt[4]{1}, \beta \in 8 \sqrt[4]{1}, \gamma \in \frac{4}{27} \sqrt[4]{2(7+i 5 \sqrt{11})^{3}}$ and $\delta \in$ $\frac{4}{27} \sqrt[4]{2(7-i 5 \sqrt{11})^{3}}$

Example 9. We have (see [7]):

$$
\begin{aligned}
f_{4}(\mathbb{X}) & =\mathbb{X}^{4}-\mathbb{X}^{3}-4 \mathbb{X}^{2}-4 \mathbb{X}+1 \\
= & {\left[\left(\mathbb{X}-2 \cos \frac{2 \pi}{15}\right)\left(\mathbb{X}-2 \cos \frac{8 \pi}{15}\right)\right]\left[\left(\mathbb{X}-2 \cos \frac{4 \pi}{15}\right)\left(\mathbb{X}-2 \cos \frac{14 \pi}{15}\right)\right] } \\
= & \left(\mathbb{X}^{2}-2\left(\cos \frac{\pi}{5}\right) \mathbb{X}+2\left(\cos \frac{\pi}{5}-1\right)\right)\left(\mathbb{X}^{2}+\left(2 \cos \frac{\pi}{5}-1\right) \mathbb{X}-1-2 \cos \frac{\pi}{5}\right) \\
& =\left(\mathbb{X}^{2}-\varphi \mathbb{X}+\varphi-2\right)\left(\mathbb{X}^{2}+(\varphi-1) \mathbb{X}-\varphi-1\right),
\end{aligned}
$$

where $\varphi:=2 \cos \frac{\pi}{5}=\frac{1+\sqrt{ } \overline{5}}{2}$ is the golden ratio and

$$
f_{4}(\mathbb{X})=0 \quad \Leftrightarrow \quad\left(\mathbb{X}^{2}-\frac{1}{2} \mathbb{X}-\frac{17}{8}\right)^{2}=\left(\frac{15}{8}\right)^{2}\left(1-\frac{8}{15} \mathbb{X}\right) .
$$

Thus, we get

$$
\begin{aligned}
\sqrt{1-\frac{16}{15} \cos \frac{2 \pi}{15}} & \sqrt{1-\frac{16}{15} \cos \frac{14 \pi}{15}} \\
& =\sqrt{1-\frac{16}{15} \cos \frac{4 \pi}{15}}+\sqrt{1-\frac{16}{15} \cos \frac{8 \pi}{15}} \\
= & \frac{4}{15}\left(\cos \frac{2 \pi}{15}-\cos \frac{8 \pi}{15}+3 \cos \frac{4 \pi}{15}-3 \cos \frac{14 \pi}{15}\right) \\
& =\frac{2}{15}\left(\sqrt{\frac{15-3 \sqrt{5}}{2}}+3 \sqrt{\frac{15+3 \sqrt{5}}{2}}\right) .
\end{aligned}
$$

We note that

$$
\cos \frac{2 \pi}{15}=\frac{1}{4}\left(\frac{1+\sqrt{5}}{2}+\sqrt{\frac{15-3 \sqrt{5}}{2}}\right)
$$




$$
\begin{aligned}
& \cos \frac{4 \pi}{15}=\frac{1}{4}\left(\frac{1-\sqrt{5}}{2}+\sqrt{\frac{15+3 \sqrt{5}}{2}}\right), \\
& \cos \frac{8 \pi}{15}=\frac{1}{4}\left(\frac{1+\sqrt{5}}{2}-\sqrt{\frac{15-3 \sqrt{5}}{2}}\right), \\
& \cos \frac{14 \pi}{15}=\frac{1}{4}\left(\frac{1-\sqrt{5}}{2}-\sqrt{\frac{15+3 \sqrt{5}}{2}}\right) .
\end{aligned}
$$

\section{Final Remark}

Our colleague from Russia Sergey Markelov informed us in private correspondence about deriving several new formulae of the type discussed in the current paper. Among others, he found the following ones

$$
\begin{aligned}
& \sqrt[3]{\sin (\boldsymbol{\alpha})}+\sqrt[3]{\sin (2 \boldsymbol{\alpha})}+\sqrt[3]{\sin (4 \boldsymbol{\alpha})} \\
&=\sqrt[3]{\frac{\sqrt[3]{7}}{3}-2+\sqrt[3]{3 \sqrt[3]{7}-4}+\sqrt[3]{3 \sqrt[3]{7}-5} \sqrt[3]{\frac{3}{2} \sqrt[6]{7}}}
\end{aligned}
$$

More information can be found in http://ru-math.livejournal.com/79774.html - the Russian internet forum where sums of this type are considered and where some efforts are made to present them in the general context.

\section{References}

[1] L. Bankoff, J. Garfunkel, The heptagonal triangle, Mathematics Magazine, 46 (1973), 7-19.

[2] Z. Čerin, Regular heptagon's intersections circles, Elemente der Mathematik, 61 (2006), 138-151.

[3] Y. Latushkin, V. Ushakov, A representation of regular subsequences of recurrent sequences, The Fibonacci Quarterly, 43 (2005), 70-84.

[4] V. Shevelev, On Ramanujan cubic polynomials, South East Asian Journal of Mathematics and Mathematical Sciences, 8 (2009), 113-122.

[5] N.J.A. Sloane, The On-Line Encyclopedia of Integer Sequences, http://oeis.org/ (2013). 
[6] R. Wituła, Full description of Ramanujan cubic polynomials, Journal of Integer Sequences, 13 (2010), article 10.5.7.

[7] R. Wituła, On Some Applications of the Formulae for Sums of Unimodular Complex Numbers, Wyd. Prac. Komput. J. Skalmierskiego, Gliwice (2011) (in Polish).

[8] R. Wituła, Ramanujan cubic polynomials of the second kind, Journal of Integer Sequences, 13 (2010), article 07.5.6.

[9] R. Wituła, Ramanujan type trigonometric formulae, Demonstratio Mathematica, 45 (2012), 779-796.

[10] R. Wituła, Ramanujan type trigonometric formulas: the general form for the argument $\frac{2 \pi}{7}$, Journal of Integer Sequences, 12 (2009), article 09.8.5.

[11] R. Wituła, D. Słota, New Ramanujan-type formulas and quasi-Fibonacci numbers of order 7, Journal of Integer Sequences, 10 (2007), article 07.5.6.

[12] R. Wituła, D. Słota, Quasi-Fibonacci numbers of order 13 on the occasion the Thirteenth International Conference on Fibonacci Numbers and Their Applications, Congressus Numerantium, 201 (2010), 89-107.

[13] R. Wituła, E. Hetmaniok, D. Słota, Sums of rational powers of the roots of cubic polynomials, In Preparation. 
